\title{
Subliminal Affective Priming of Internet Emotions Influences Attentional Bias
}

\author{
Jie Cheng \\ School of Education, Tianjin University \\ Tianjin, China \\ 18404902078@163.com
}

\begin{abstract}
This research aims at studying the effect of subliminal affective priming of Internet emotions on the attentional bias. The prime stimulus of this experiment are Internet emotions, which take from a social networking software. The experiment adopts a single factor within-subject design. The independent variable is the emotional valence of Internet emotions (positive, neutral, negative). The dot-probe paradigm is used to examine the differences of reaction time to the probe stimulus. The results show that subliminal presence of positive Internet emotions do not affect the attentional bias. Subliminal presence of negative Internet emotions would influence the attentional bias. There is a significant faster reaction time to negative pictures than to neutral pictures after the negative affective priming of Internet emotions. The subliminal presence of the neutral Internet emotions do not affect attentional bias.
\end{abstract}

Keywords-Internet emotions; Subliminal affective priming; Dot-probe paradigm; Attentional bias

\section{INTRODUCTION}

\section{A. Internet emoticons}

With the development of the Internet nowadays, people are increasingly unable to live a normal life without social software such as mobile QQ and WeChat. In addition to using for communication, Internet emoticons are also deeply loved by people because of their intuitiveness, vividness, and fun. By evaluating the emotional valence of Internet emoticons, two main emotional dimensions of valence and arousal were extracted. It indicates that the Internet emoticons have emotional characteristics and can be used as emotional priming materials during research. Researchers have found that online emoticons have the effect of communicating information that resembles the true expression of daily life. They would influnce emotions and attitude judgments. Zhang Xiaowei studied the subliminal affective priming of Internet emoticons. The results showed that Internet emoticons can generate subliminal emotional priming effect.

\section{B. Subliminal affective priming}

Emotional priming effect has two meanings. On the one hand, it means that the emotional valence of probe stimulus is consistent with the prime stimulus, and the reaction time of the participants will be shorter and the accuracy rate will be improved under the consistent condition. On the other hand, the emotional characteristics of the prime stimulus will affect the subsequent cognitive processing. Studies have shown that when the presentative time of prime stimulus is extremely brief and below the sensory threshold, the affective priming effect can still be observed. This phenomenon is known as "Subliminal affective priming".

\section{Attentional bias}

Attentional bias means that individuals with a high degree of sensitivity to specific stimulus and generate selective attention. This selective attention will make limited attention resource occupied, so that other stimulus information can not be processed.

\section{The relationship between affective priming and attention} bias

When there are plenty of different kinds of dangers in the environment, individuals will make adaptive responses to specific situations or stimuluses, which is conform to the trend of human evolution. According to N.Kyle Smith, when the individual is dominated by a negative emotion model, compared to the positive stimulus, the negative stimulus will have higher accessibility, so the individual is more likely to show attentional bias to the negative ones. When the positive model is in a dominant position, individuals will allocate more attention resources to positive events.

\section{E. Negative attention bias}

For external stimulus that give rise to unpleasant emotions, such as bloody violent images and furious expressions, individuals will give priority in cognitive processing and behavioral responses. This is known as negative emotion bias. The study have showed that groups with anxiety disorder or high anxiety appear attentional bias toward threatening stimuluses. In other words, they response more quickly to negative stimuluses than to neutral stimuluses. However, whether the nonanxious individuals also show attention bias to negative stimuluses, there are still different conclusions in the present research findings. 
Based on the previous studies, the study uses Internet emotions as prime stimulus and dot-probe paradigm as experimental paradigm to further study the influence of subliminal affective priming of Internet emotions on attentional bias.

\section{METHOD}

\section{A. Participants}

Participants are undergraduate students in Shanxi Datong University. Before the experiment, participants were asked to write self-rating anxiety scale and through the screening, 58 non-anxiety participants were finally obtained. There are 19 boys and 39 girls.

\section{B. Experimental paradigm}

The dot-probe paradigm was adopted in this experimented experimental procedure is at first, a series of paired stimuluses, i.e., emotional stimulus and neutral stimulus appears in the screen. After the presentation is completed, the probe stimulus, i.e., a dot will appear immediately in one of the paired stimuluses positions. Then we can know the attentional bias of participants through the speed of the button response. The paradigm includes two conditions of consistency and inconsistency. When the position of the probe stimulus is the same as emotional stimulus, it is called the consistent condition. When the position of the probe stimulus is the same as the neutral stimulus, it is called the inconsistent condition. In general, the reaction time of the consistent condition is significantly faster than the inconsistent condition.

\section{Experimental design}

A single factor within-subject design was adopted in this experiment. The independent variable was the emotional valence of prime stimulus (positive, neutral, negative). At each level of the independent variable, there were consistent and inconsistent conditions. After presenting a positive Internet emotional picture, we will compare the reaction time differences of the probe stimulus on the position of positive picture and the position of neutral picture. Similarly, we will compare the reaction time differences of the probe stimulus on the position of negative picture and neutral picture after a negative Internet emotional picture. Finally, we will make a comparison between the probe stimulus on the position of emotional picture and neutral picture after a neutral Internet emotional image.

\section{Materials}

SAS: The Anxiety Self-Assessment Scale has wide applicability and is mainly used to assess the subjective sensibility of anxious patients. It contains 20 items. Each item has four options. "1", "2", “3", "4" respectively means: completely, somewhat, moderately, and very clearly. Item 5, 9, 13, 17, and 19 scores reversely. After getting a total score and multiply by 1.25 to get a new number, and then remove the decimal point of the number, which is a final score. According to the norm, score of 50 and below are not anxious and are valid experimental data.
Prime stimulus materials: The prime stimulus for the experiment were 90 Internet emotional pictures which were randomly selected from a social software. According to make a 7-rank grade to these pictures by 20 students from the department of Psychology of Datong University, based on two dimensions of valence and arousal,20 pictures of positive, negative and neutral were respectively selected.

There was a significant difference in the degree of valence between the three groups of Internet emotions $(\mathrm{F}=147.58$, $p<0.01)$. Further comparison results showed that the positive emotional pictures were significantly more pleasant than the neutral emotional pictures, and the neutral emotional pictures were significantly more pleasant than negative emotional pictures. The $\mathrm{p}$ values were both less than 0.01 (see Table 1). There was no significant difference in arousal between the three groups of Internet emotions $(\mathrm{F}=0.29, p>0.05)$.

TABLE I ThE VALENCE AND ARousal of DifFERENT EMotional PICTURES

\begin{tabular}{ccc}
\hline Type & Valence $(M \pm S D)$ & Arousal $(M \pm S D)$ \\
\hline Positive & $5.71 \pm 0.76$ & $4.05 \pm 0.31$ \\
Neutral & $3.60 \pm 0.39$ & $4.01 \pm 0.20$ \\
Negative & $2.13 \pm 0.76$ & $3.97 \pm 0.18$ \\
\hline
\end{tabular}

Picture Materials: The pictures before probe stimulus choose from the Picture Library of International Emotions (IAPS), including 30 positive, 30 negative and 60 neutral emotional pictures.

\section{E. Procedure}

The experimental task is presented by E-prime 2.0. There are 3 blocks in total. There are 20 trails in each block. So there are a total of 60 trails, which are presented randomly. Five trails are practiced before the formal experiment. Participants can rest twice during the experiment, and the duration of rest will be determined by the participants.

In each trail, "+" of $800 \mathrm{~ms}$ will appear in the center of the screen, then there will be a blank of $500 \mathrm{~ms}$, and a $40 \mathrm{~ms}$ prime stimulus will appear. After the $200 \mathrm{~ms}$ interval, there will be a pair of pictures appear with form of left and right which last $1100 \mathrm{~ms}$. Following the pictures, the probe stimulus "." is presented. The task of the participant is to accurately and quickly make a key reaction after seeing the dot. If the dot appears on the left picture, press the " $F$ " key; if the dot appears on the right picture, press "J" key. The program will enter the next trail automatically and record the reaction time. 


\section{RESULTS}

\section{A. The Difference Tests of reaction time after positive Internet emotions}

The results(see Table 2) show that after subliminal presence of positive Internet emotions, the reaction time of the probe stimulus appearing in the neutral picture position (inconsistent) is faster than that in the positive picture position (consistent), but the difference was not significant $(\mathrm{t}=1.61$, $p>0.05)$.

TABLE II PAIRED SAMPLE T-TeSt OF REACTION TIME

\begin{tabular}{cccc}
\hline Condition & $\mathrm{M} \pm \mathrm{SD}$ & $\mathrm{t}$ & $p$ \\
\hline Consistency & $470.58 \pm 103.22$ & & \\
Inconsistency & $454.12 \pm 85.73$ & 1.61 & 0.11 \\
\hline
\end{tabular}

B. The Difference Tests of reaction time after negative Internet emotions

From the results in Table 3, it indicates that after presence of negative Internet emotions, the reaction time of the probe point appears in the position of the negative picture (consistent) is faster than the position of the neutral picture (inconsistent), and the difference is significant $(\mathrm{t}=2.94, p<0.05)$.

TABLE III Paired SAmple T-Test of Reaction Time

\begin{tabular}{cccc}
\hline Condition & $\mathrm{M} \pm \mathrm{SD}$ & $\mathrm{t}$ & $p$ \\
\hline Consistency & $463.54 \pm 86.78$ & 2.94 & 0.00 \\
Inconsistency & $478.38 \pm 103.59$ & & \\
\hline
\end{tabular}

\section{The Difference Tests of reaction time after neutral Internet emotions}

From the results in Table 4, it can be seen that after presence of neutral Internet emotions, the reaction time of the probe point appears in the position in the neutral picture position is the fastest, and in the positive picture position is the slowest, but the difference is not significant ( $\mathrm{F}=0.38, p>0.05)$.

TABLE IV ONE-QAY ANOVA OF REACTION TIME

\begin{tabular}{cccc}
\hline Type & $\mathrm{M} \pm \mathrm{SD}$ & $\mathrm{F}$ & $p$ \\
\hline Positive & $482.20 \pm 110.76$ & & \\
Neutral & $472.49 \pm 86.64$ & 0.38 & 0.68 \\
Negative & $466.30 \pm 97.90$ & & \\
\hline
\end{tabular}


the interference of negative events and concentrate more attention on current task.

\section{CONCLUSION}

The conclusion of this study is that subliminal presence of positive Intenet emotions do not affect the attentional bias. Subliminal presence of negative Intenet emotional pictures would influence the attentional bias. There is a significant faster reaction time to negative images than to neutral after the negative affective priming. The presentation of the neutral Internet emotional images under the threshold does not affect attentional bias.

\section{REFERENCES}

[1] Qin Minhui, Zhou Zhuozhao, Zhongyiping. Subliminal Affective Priming of Internet Emotions Influences Cognition Bias [J]. Psychological Research,2015,8(3):46-50.

[2] Chen Qipeng.Initial Assessment of Internet Symbols Affective Valence [D].Chang sha:HunanNormal University,2008.

[3] Zhang Xiaowei,Liu Dan,Wang Hongyi.A Study on the Subliminal Affective Priming Effect by Network Expressions [D]. Beijing: Beijing Sport University,2012.

[4] Lv Yong, Zhang Weina, Shen Deli. Subliminal Affective Priming Effect by Faces With Different Valence: An ERP Study[J]. Acta Psychologica Sinica, 2010,42(9):929-938.

[5] Peng Xiaozhe, Zhou Xiaolin.Emotional Information and Attentional Bias [J]. Advances in Psychological Science, 2005, 13 (4): 488-496.

[6] N.Kyle Smith, Jeff T.Being Bad isn' t Always Good: Affective Context Negative Information [J]. Joumal of Personality and Social Psychology, 2006, 90(2):210-220.

[7] Song Jia. The relationship between emotions evoked and attentional bias of undergraduate [D]. Kunming: Yunnan Normal University, 2014.

[8] Koster, Crombez G,Verschuere B.Selective attention to threat in the dot probe paradigm:Differentiating vigilance and difficulty to disengage[J]. Behaviour Research and Therapy, 2004,42(10):1183-1192.

[9] Duan Quanquan,Sheng Li.Differential validity of SAS and SDS among psychiatric non-psychotic outpatients and their partners[J]. Chinese Mental Health Journal, 2012,26 (9):676-679.

[10] Zhang Dongdong.Experimental research of emotional priming's regulating role of attentional bias [D]. Beijing: Capital Normal University, 2009. 